\title{
Gamma signatures of the C-BORD Tagged Neutron Inspection System
}

\author{
A. Sardet, B. Pérot, C. Carasco, G. Sannié, S. Moretto, G. Nebbia, C. Fontana, F. Pino, A. Iovene, \\ C. Tintori
}

\begin{abstract}
In the frame of C-BORD project (H2020 program of the EU), a Rapidly relocatable Tagged Neutron Inspection System (RRTNIS) is being developed to non-intrusively detect explosives, chemical threats, and other illicit goods in cargo containers. Material identification is performed through gamma spectroscopy, using twenty $\mathrm{NaI}$ detectors and four $\mathrm{LaBr}_{3}$ detectors, to determine the different elements composing the inspected item from their specific gamma signatures induced by fast neutrons. This is performed using an unfolding algorithm to decompose the energy spectrum of a suspect item, selected by $\mathrm{X}$ ray radiography and on which the RRTNIS inspection is focused, on a database of pure element gamma signatures. This paper reports on simulated signatures for the $\mathrm{NaI}$ and $\mathrm{LaBr}_{3}$ detectors, constructed using the MCNP6 code. First experimental spectra of a few elements of interest are also presented.
\end{abstract}

Index Terms-C-BORD project, Rapidly Relocatable Tagged Neutron Inspection System, fast neutron activation analysis, inelastic scattering gamma rays, MCNP6

\section{INTRODUCTION}

G LOBALIZATION and the increase of trade is accompanied by an increase in the traffic of dangerous substances (such as explosives, illicit drugs, etc.) but also in contraband. Each year, about 4 billion tons of freight is arriving at the European border and the inspection of all these cargo is not compatible with economic constraints. Therefore, the effective Container inspection at BORDer control points (C-BORD) project, launched in the frame of $\mathrm{H} 2020$ European projects for the development of "Technologies for inspections of large volume freight", aims at developing a toolbox of five nonintrusive inspection technologies, which, working in combination, would ensure a more efficient screening of cargo containers. Among the developed techniques, the Rapidly Relocatable Tagged Neutron Inspection System (RRTNIS) is

Manuscript received June 30, 2017. This work has received funding from the European Union's Horizon 2020 research and innovation program under grant agreement No 653323. This text reflects only the author's views and the Commission is not liable for any use that may be made of the information contained therein.

A. Sardet, B. Pérot and C. Carasco are with CEA, DEN, Cadarache, Nuclear Measurement Laboratory, F-13108 Saint-Paul-lez-Durance, France (email: alix.sardet@cea.fr).

G. Sannié is with CEA, DRT, LIST, Saclay, F-91191 Gif-Sur-Yvette, France.

S. Moretto, G. Nebbia, C. Fontana and F. Pino are with Dipartimento di Fisica e Astronomia e INFN di Padova, via Marzolo, 8, I-35131 Padova, Italy.

A. Iovene and C. Tintori are with CAEN S.p.A, Via Vetraia, 11, I-55049 Viareggio, Italy. designed to be a second-line defense system, which would help identify threats and contraband materials in localized parts of the container, following the identification of a suspect item by X-ray radiography. After a brief presentation of the design of the system and its operation mode, the method used to build simulated elemental gamma spectra is detailed. Finally, the experimental setup used to measure these gamma signatures is presented and first measured spectra are reported.

\section{The RAPIDLy Relocatable TAGged NEUtRon INSPECTION SYSTEM}

Fig. 1 presents a schematic view of the system designed in the scope of the C-BORD project [1].

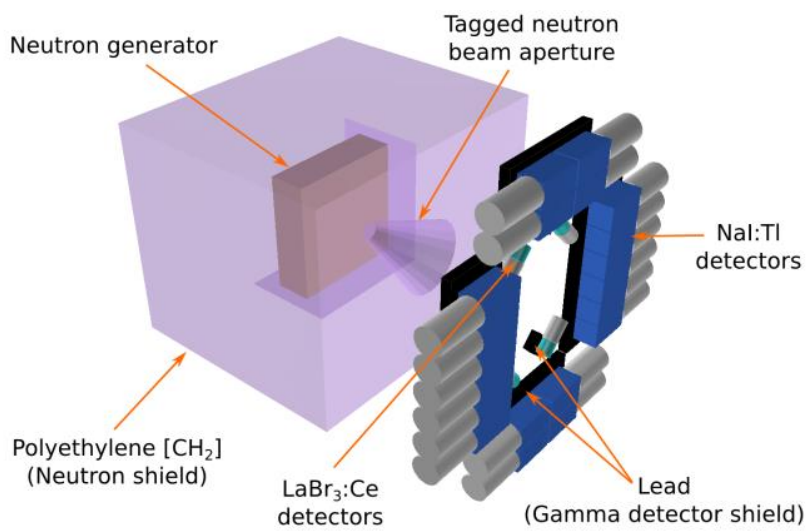

Fig. 1: Schematic view of the RRTNIS of the C-BORD project

A neutron generator, encased in a polyethylene shield to ensure radiation protection, is used to produce $14 \mathrm{MeV}$ neutrons, through the $d+t \rightarrow n+\alpha$ fusion reaction. As the alpha particle is emitted almost back-to-back with the neutron, the use of a position sensitive YAP scintillator enables a spatial selection of the interrogating fast neutrons. In C-BORD RRTNIS, a $20^{\circ}$ tagged neutron cone has been chosen, corresponding to the tagged neutron beam aperture shown in Fig. 1. In addition, the coincidence time between the alpha and the neutron-induced gamma detections allows determining the neutron time of flight, and subsequently its distance to interaction. By combining information on the direction of the neutron and its traveled distance, the gamma spectrum of any elementary volume (voxel) in the container can be reconstructed (see further in Fig. 2).

Gamma rays resulting from tagged neutron interactions are detected by twenty $5 " \times 5 " \times 10 "$ parallelepiped-shaped NaI 
detectors and four $3 " \times 3$ " cylindrical $\mathrm{LaBr}_{3}$ detectors placed in a backscatter position, near the neutron generator. The large $\mathrm{NaI}$ scintillators are used to detect gamma rays above $600 \mathrm{keV}$, which allows identifying most common elements such as carbon, nitrogen, oxygen, silicon, chlorine, calcium, aluminum, iron, chromium, nickel, copper, zinc, lead, etc. The high resolution $\mathrm{LaBr}_{3}$ scintillators are mainly used to detect gamma rays below $500 \mathrm{keV}$, in view to identify some of the chemical warfare elements that do not show clear gamma signatures at higher energy, such as arsenic, bromine, and iodine. A $5 \mathrm{~cm}$ thick lead plate is used to shield all gamma detectors from scattered neutrons and gamma rays coming from the polyethylene shield.

All elements of the system (neutron generator, gamma detectors, shields, electronics, etc.) are placed on the same side of the container in a $270 \times 250 \times 220 \mathrm{~cm}^{3}$ box, fixed on a lifting platform so as to be able to inspect the cargo container at different heights. The total weight of the system is estimated to be of about 5 tons.

The gamma spectrum obtained by summing the spectra of

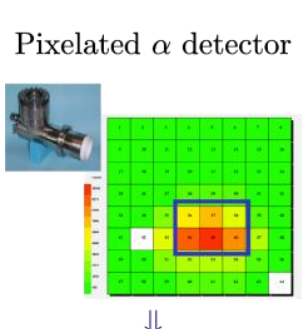

Neutron direction
Neutron time of flight spectrum

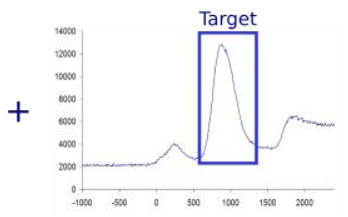

$\Downarrow$

Distance traveled the $\mathrm{NaI}$ or $\mathrm{LaBr}_{3}$ detectors results from individual gamma signatures of the interrogated elements, weighted by their chemical proportions in the cargo materials. It can be fitted by a linear combination of these individual signatures, using an unfolding algorithm and a database of pure element gamma signatures (see Fig. 2). The retrieved weights give information on the chemical composition of the target, thus allowing its identification. For instance, the target can be categorized as metallic, ceramic or organic. For this last category, additional information is obtained by looking at the proportions of carbon $(\mathrm{C})$, nitrogen $(\mathrm{N})$ and oxygen $(\mathrm{O})$, using a barycentric representation as shown in Fig. 2 (triangle). Unfolding the whole gamma spectrum applies well for the identification of most common elements, but for those having gamma signatures only at low energy, such as arsenic, bromine, or iodine, identification is mainly based on peak detection in the $\mathrm{LaBr}_{3}$ spectrum.

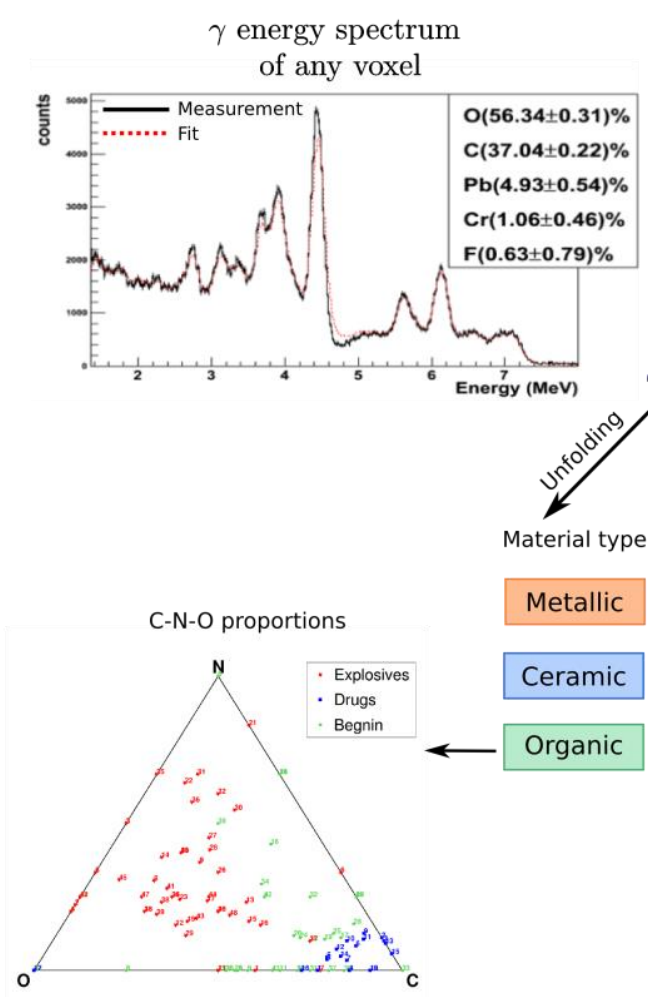

Elementary gamma signature database

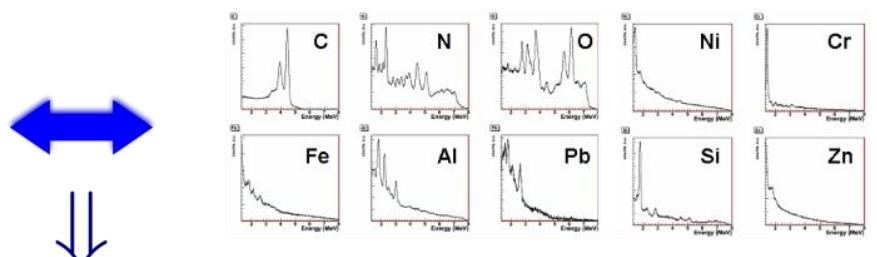

Target identification
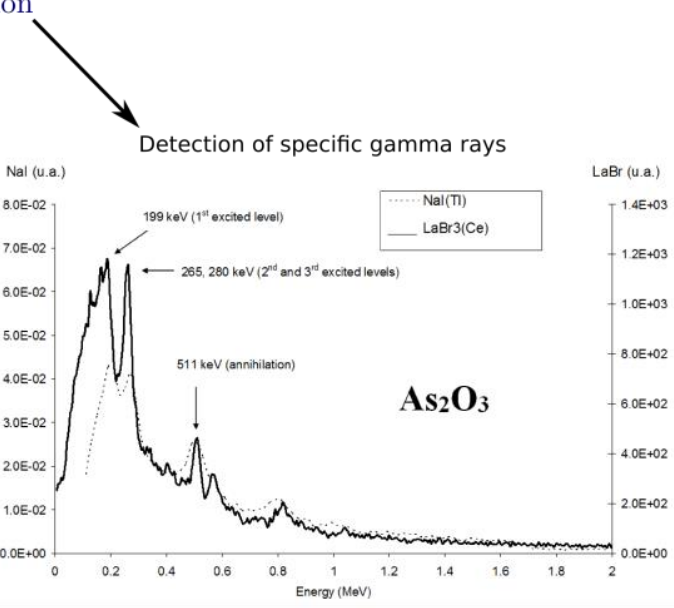

Fig. 2: Data analysis principle of the RRTNIS. 


\section{SIMULATION OF NEUTRON-INDUCED GAMMA SPECTRA}

In order to assess the performances of different unfolding algorithms developed by C-BORD partners, simulated pure element gamma signatures have been generated for both $\mathrm{NaI}$ and $\mathrm{LaBr}_{3}$ detectors, using the MCNP6 code. The interrogation of a pure element target by the RRTNIS was modeled with MCNP6 [2] as presented in Fig. 3.
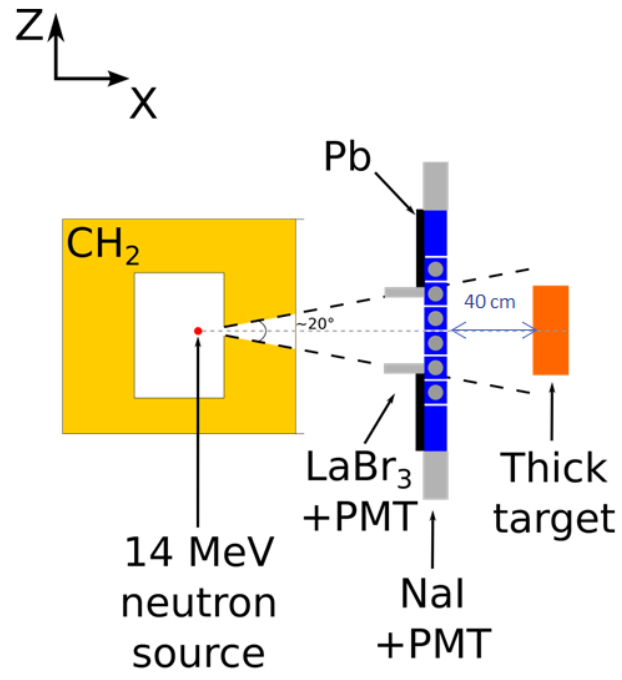

\section{Concrete}

Fig. 3: MCNP6 model of a pure element target inspection, for the construction of the RRTNIS simulated gamma signature database.
The associated particle neutron generator is assimilated to a point-like $14 \mathrm{MeV}$ neutron source with a $20^{\circ}$ conical emission. The floor is modeled by a $100 \mathrm{~cm}$ thick layer of concrete and the target consists of a $20 \times 50 \times 50 \mathrm{~cm}^{3}$ parallelepiped of the pure element of interest, with a density of $1 \mathrm{~g} / \mathrm{cm}^{3}$. The MCNP6 point detector (F5 tally) is used in the front face of each detector to estimate the incoming photon flux. This last is then processed by MODAR software [3] to account for detection efficiency, based on MCNP calculations of the energy deposition in $\mathrm{NaI}$ or $\mathrm{LaBr}_{3}$ crystals (F8 pulse height tally), and for time and energy broadening due to the resolution of the detectors taken from [4] [5]. An example of this procedure is presented in Fig. 4 for a thick carbon target.
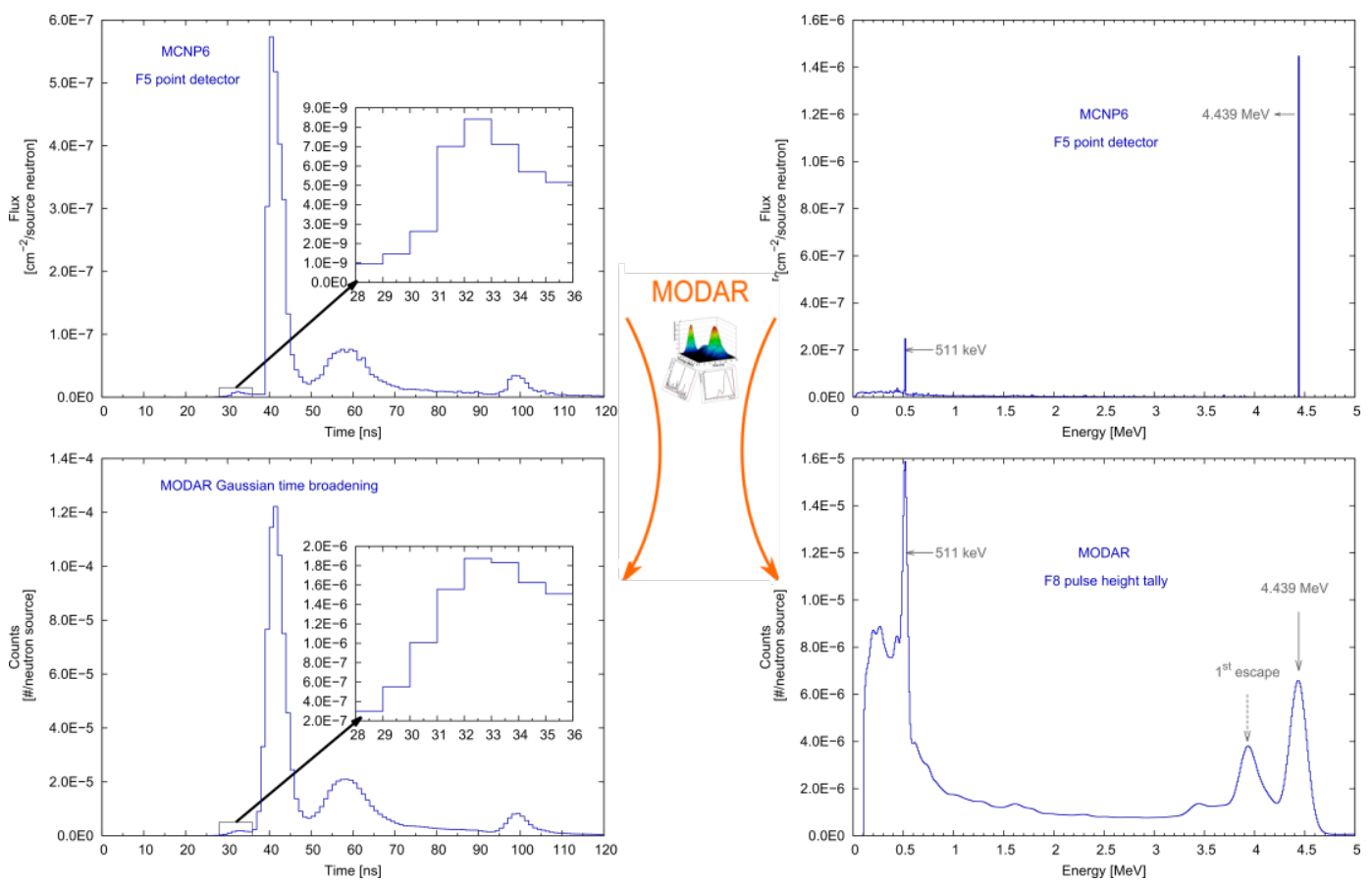

Fig. 4: Conversion of the photon flux reaching a 5" 5 " $\times 10$ " NaI detector into TOF (left) and gamma (right) spectra using MODAR, in case of a carbon target.

The time-of-flight (TOF) spectrum of each detector can then be converted into a distance spectrum using the $\sim 5 \mathrm{~cm} \cdot \mathrm{ns}^{-1}$ speed of $14 \mathrm{MeV}$ neutrons, and the spectra of all $\mathrm{NaI}$ or $\mathrm{LaBr}_{3}$ detectors can be summed to improve statistics. The gamma spectrum of the region of interest can then be plotted by selecting the corresponding spatial (or TOF) region, as can be seen in Fig. 5 for a carbon target.

Note that MODAR can also be used to process experimental data, in which case time selection allows suppressing random coincidences (see further in Fig. 9), which are not simulated here. 

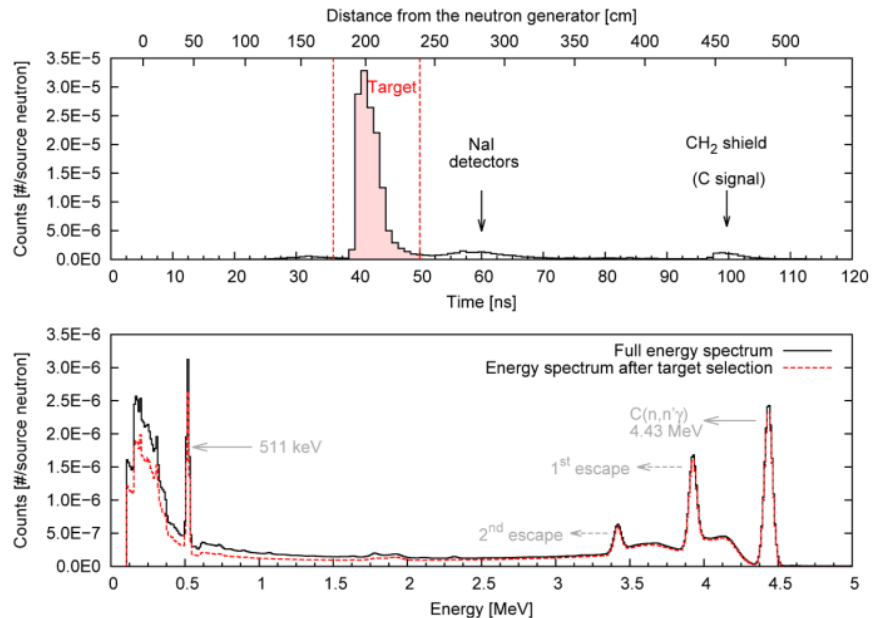

Fig. 5: Time of flight spectrum (upper panel) and energy spectrum (lower panel) of a $\mathrm{LaBr}_{3}$ detector for a thick carbon target. The gamma spectrum presented with a dashed red line was obtained after a time of flight selection of the target. The total spectrum without selection is plotted with the black full line.

Two databases of gamma signatures have been constructed for the $\mathrm{NaI}$ and $\mathrm{LaBr}_{3}$ detectors, respectively. An extract of the $\mathrm{NaI}$ database is presented in Fig. 6.
As mentioned above, $\mathrm{LaBr}_{3}$ detectors will mainly be used to detect specific gamma rays, below $500 \mathrm{MeV}$, signing the presence of chemical warfare agents such as arsenic which do not exhibit gamma signatures at higher energy (see Fig. 7).

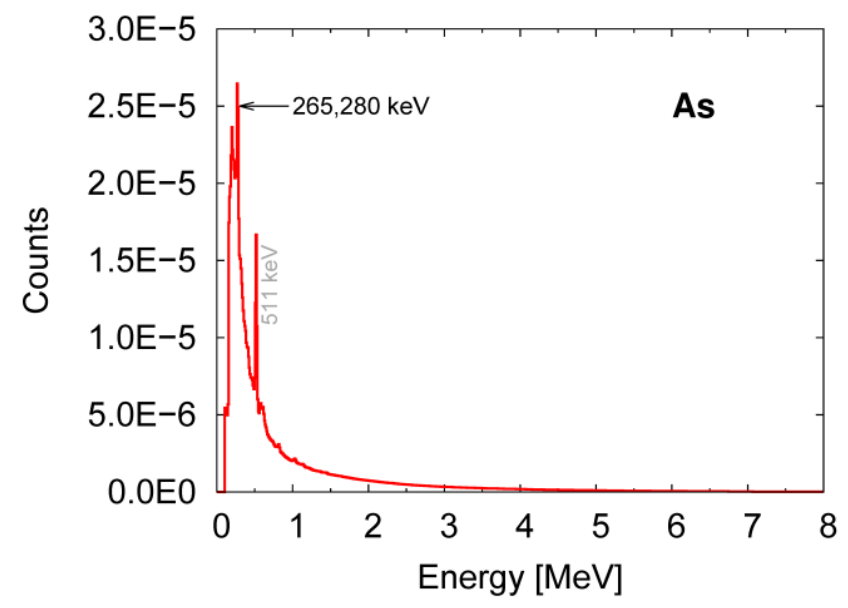

Fig. 7: Simulated gamma signature of arsenic in a $\mathrm{Labr}_{3}$ detector.
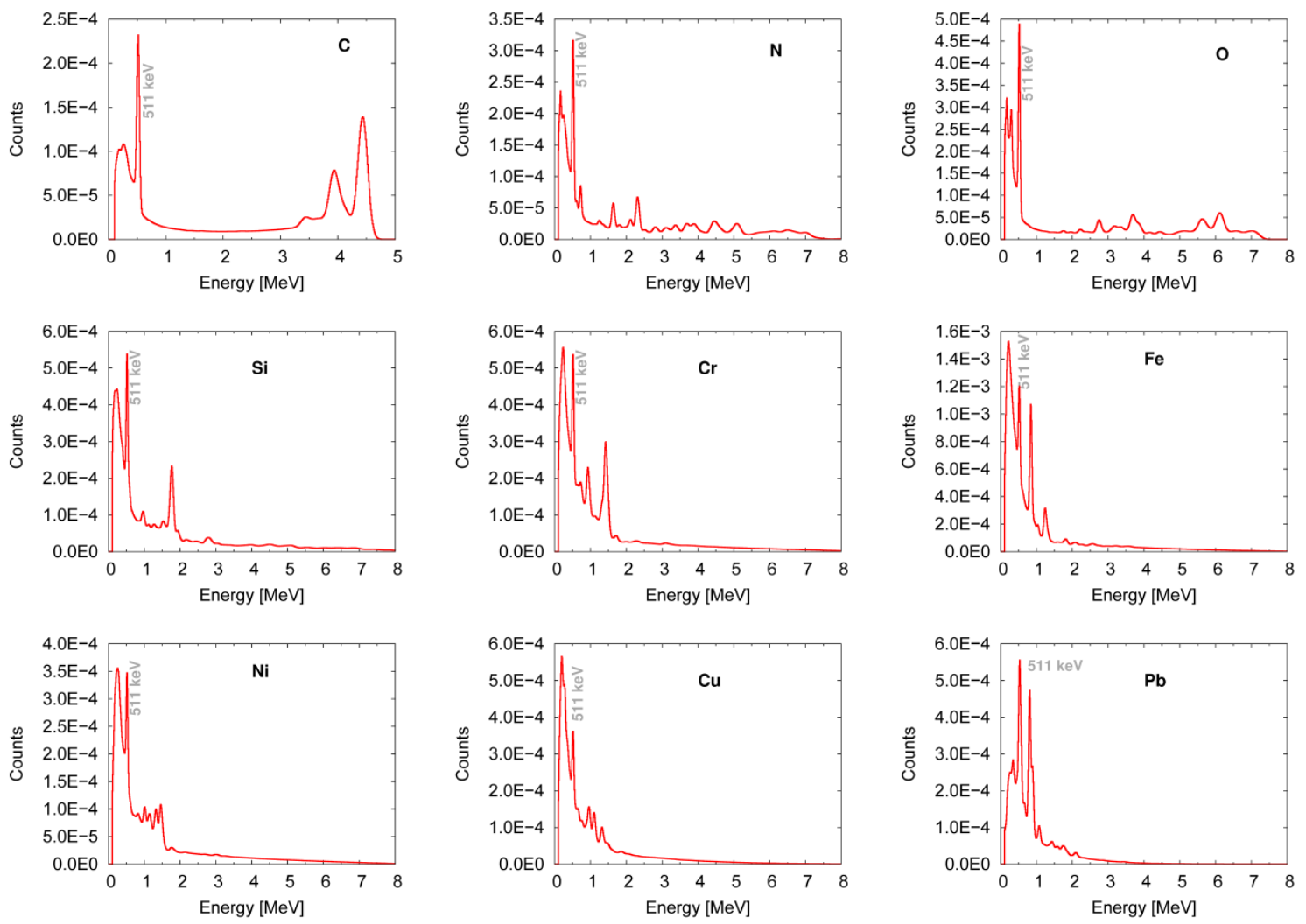

Fig. 6: Simulated gamma signatures of common elements in NaI detectors. 


\section{ACQUISITION OF EXPERIMENTAL GAMMA SIGNATURES}

Experience from past European projects EURITRACK [6] [7], Eritr@ck and UNCOSS [8] [9], showed that simulation does not entirely reproduce measured gamma signatures. For most common elements, a rather good agreement is observed, even if missing peaks or differences in the continuum can be noted. However, for unusual elements like some of those composing chemical warfare agents, unrealistic spectra can be produced by simulation when unrealistic nuclear models are used by MCNP instead of missing nuclear data.

Therefore, experimental gamma spectra are currently being acquired at CEA Cadarache, in DANAIDES irradiation cell of TOTEM nuclear facility. A picture of the experimental setup is presented in Fig. 8.

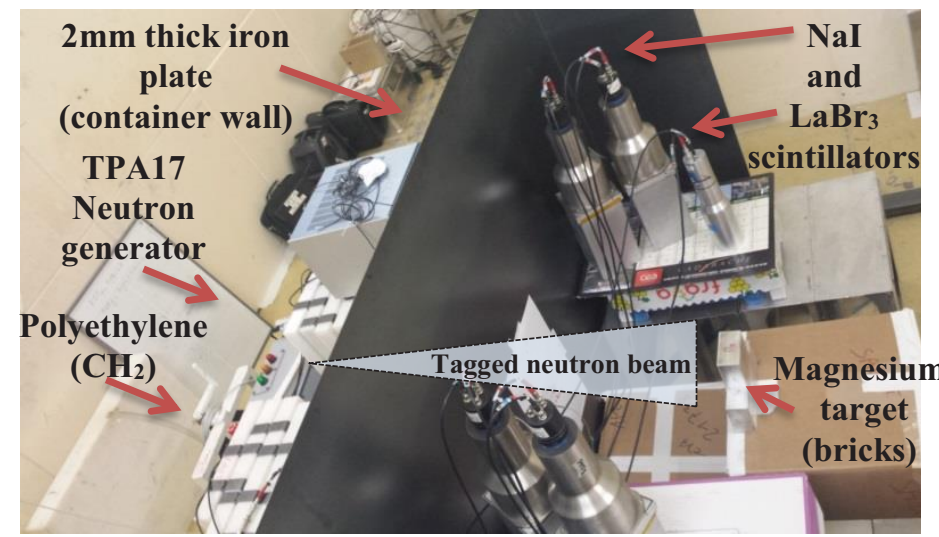

Fig. 8: Picture of the experimental setup to acquire pure element gamma signatures at CEA Cadarache. A $5 \mathrm{~cm}$ thick lead screen (not visible in the picture, between polyethylene and the iron wall) is used to shield gamma detectors.

A target of pure element (e.g. magnesium in Fig. 8) is placed in front of the tagged neutron beam produced by a TPA17 neutron generator from SODERN, similar to the one that will be used in C-BORD project. Signals from the embedded YAP detector and of the gamma detectors are processed and recorded using a digital acquisition system and raw data postprocessing software, developed in a joint effort by CAEN and UNIPD [10].

From this initial post-processed data, other software developed by CEA Cadarache is used to generate, for each detector, a correlation between the neutron TOF and the deposited gamma energy in the detector. A TOF (or spatial) selection on the target is then performed, as shown in Fig. 9 for a graphite target, to obtain the experimental gamma signature of the interrogated material.
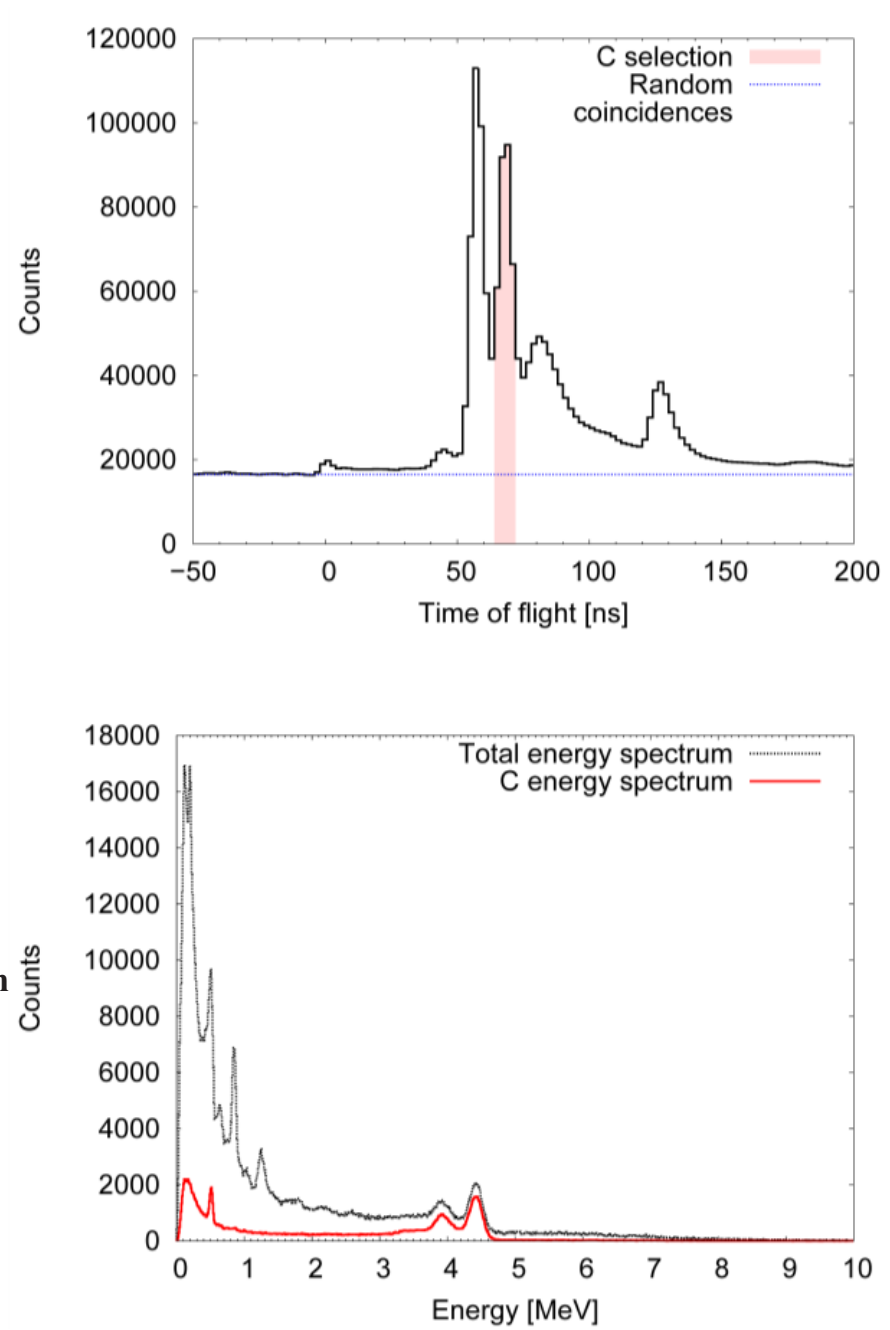

Fig. 9: Time of flight spectrum (upper panel) and energy spectrum (lower panel) on a NaI detector for a graphite target. The gamma spectrum presented with a full red line was obtained after a time of flight selection of the target and after suppression of random coincidences. The total spectrum without time selection is plotted with the black dashed line.

Fig. 10 presents experimental gamma signatures for carbon, nitrogen and oxygen recorded with the NaI detectors. 

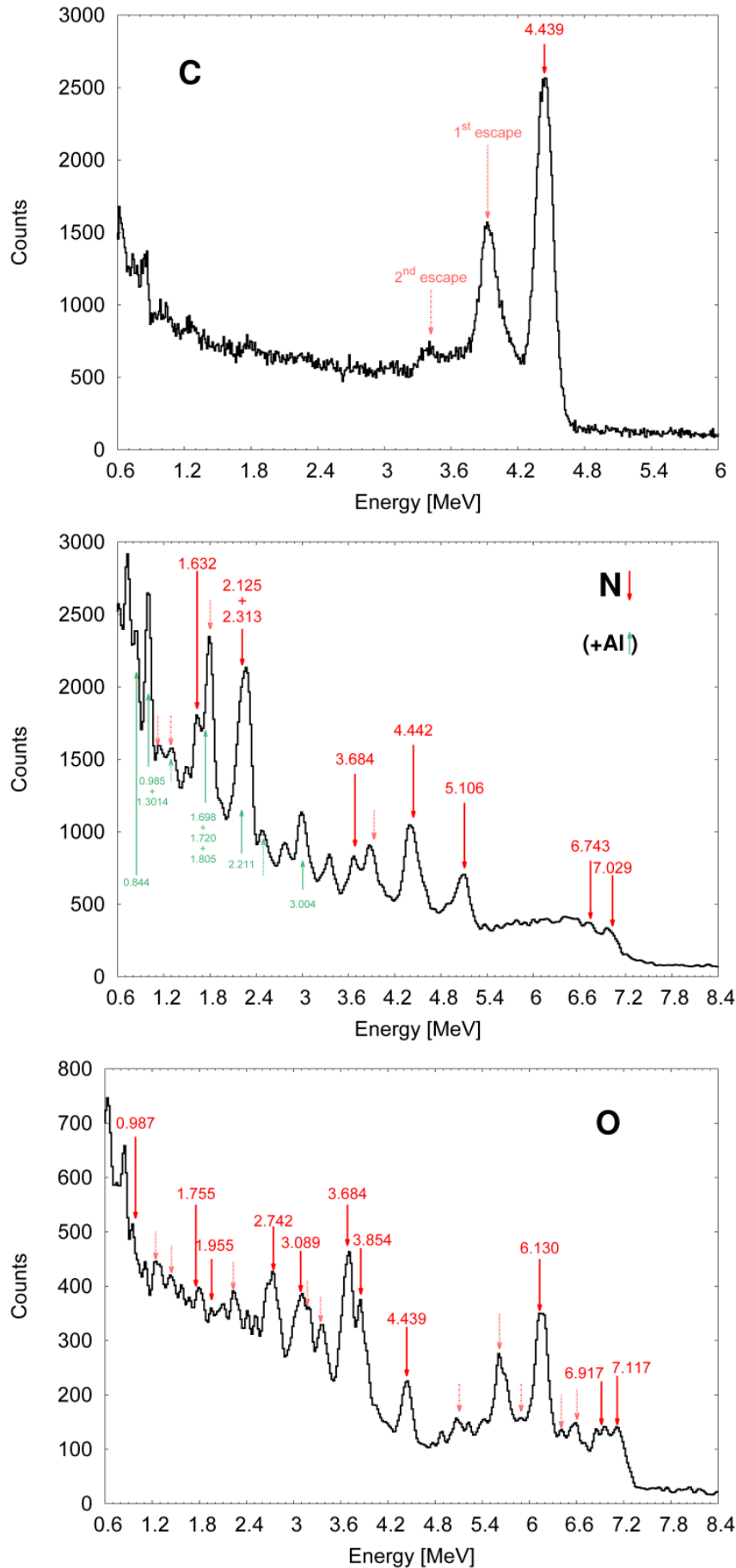

Fig. 10: Experimental gamma signatures of carbon (top), nitrogen (middle) and oxygen (bottom). Dashed arrows are used to point at peaks corresponding to first escapes, while dotted arrows are used for second escapes. On the nitrogen spectrum, the aluminum signature due to the cryogenic storage Dewar has not been subtracted yet (green up arrows).

\section{CONCLUSION}

In view to decompose the $\mathrm{NaI}$ and $\mathrm{LaBr}_{3}$ gamma spectra of $\mathrm{C}$ BORD Rapidly Relocatable Tagged Neutron Inspection System (RRTNIS) into a weighted sum of elemental spectra, pure element gamma signatures are needed. Therefore, neutron-induced gamma spectra of elements of interest for cargo container inspection have been simulated with MCNP6
Monte Carlo code and post processed with MODAR software. In addition, as differences have been observed in the past between simulation and experimental spectra, such as missing peaks or significant differences in the continuum, the acquisition of neutron-induced gamma signatures is underway at CEA Cadarache. This database of gamma spectra will be used to identify the interrogated materials during RRTNIS tests, first in the laboratory in JRC Ispra (Italy) and CEA Saclay (France) for the commissioning of the system, in 2017, and then for in-situ demonstrations at DCT Gdansk (Poland) and DCA Rotterdam (The Netherlands), in 2018.

\section{REFERENCES}

[1] A. Sardet, B. Perot, C. Carasco, G. Sannié, S. Moretto, G. Nebbia, C. Fontana, M. Moszynski, P. Sibczynski, K. Grodzicki, L. Swiderski, A. Iovene and C. Tintori, Design of the Rapidly Relocatable Tagged Neutron Inspection System of the C-BORD project, in IEEE Conference Records Nuclear Science Symposium, Strasbourg, France, 2016.

[2] Los Alamos National Laboratory, Monte Carlo N-Particle eXtended, 2008. Available: https ://mcnpx.lanl.gov/.

[3] C. Carasco, MCNP Output Data Analysis with ROOT (MODAR), Computer Physics Communications, vol. 181, pp. 1161-1666, 2010.

[4] M. Gierlik, T. Batsch, M. Moszynski, T. Szczesniak, D. Wolski, W. Klamra, B. Perot et G. Perret, Comparative study of large $\mathrm{NaI}(\mathrm{Tl})$ and BGO scintillators for the EURopean Illicit TRAfficking Countermeasures Kit project, IEEE transactions on nuclear science, vol. 53, $\mathrm{n}^{\circ} 13, \mathrm{pp}$. 1737-1743, 2006.

[5] C. Eleon, B. Perot et C. Carasco, MCNP simulations for the neutron sensor design and gamma detector selection, UNCOSS project Deliverable 3.1 \& 3.2, 2009.

[6] W. El Kanawati, B. Pérot, C. Carasco, C. Eleon, V. Valkovic, D. Sudac, J. Obhodas and G. Sannié, Acquisition of prompt gamma-ray spectra induced by $14 \mathrm{MeV}$ neutrons and comparison with Monte Carlo simulations, Applied Radiation and Isotope, no. 69, pp. 732-743, 2011.

[7] B. Pérot, C. Carasco et al., Measurement of $14 \mathrm{MeV}$ neutron-induced prompt gamma-ray spectra from 15 elements found in cargo containers, Applied Radiation and Isotopes, $\mathrm{n}^{\circ} 166, \mathrm{pp}$. 421-434, 2008.

[8] C. Eleon, B. Perot, C. Carasco, D. Sudac, J. Obhodas et V. Valkovic, Experimental and MCNP simulated gamma-ray spectra for the UNCOSS neutron-based explosive detector, Nuclear Instruments and Methods in Physics Research Section A, vol. 629, pp. 220-229, 2011.

[9] B. Pérot, C. Carasco, R. Vogler, E. Cusset, J.-L. Ma, P. Pras, M. Gmar, G. Sannié and S. Normand, Acquisition of neutron-induced gamma signatures of chemical agents, in Proceedings of the International Topical Meeting on Nuclear Research Applications and Utilization of Accelerators, IAEA, Vienna, Austria, 4-8 May 2009. Proceedings CD Series $C N-173$, http://wwwpub.iaea.org/MTCD/publications/PDF/P1433_CD/datasets/papers/sm_en07.pdf, 2009.

[10] C. L. Fontana, A. Carnera, M. Lunardon, F. Pino, C. Sada, F. Soramel, L. Stevanato, G. Nebbia, C. Carasco, B. Pérot, A. Sardet, G. Sannié, A. Iovene, C. Tintori, K. Grodzicki, M. Moszyński, P. Sibczyński, L. Swiderski and S. Moretto, "Detection System of the First Rapidly Relocatable Tagged Neutron Inspection System (RRTNIS), Developed in the Framework of the European H2020 C-BORD Project," in CAARI proceedings, Physics Procedia, 2016. 